\title{
Antimicrobial Activity of Trifoliate Orange (Poncirus trifoliate) Seed Extracts on Gram-Negative Food-bome Pathogens
}

\author{
- Research Note - \\ Seong Yeong Kim ${ }^{1}$ and Kwang-Soon $\operatorname{Shin}^{2 \dagger}$ \\ ${ }^{1}$ Nutrition Education, Graduate School of Education, and ${ }^{2}$ Department of Food Science and \\ Biotechnology, Kyonggi University, Gyeonggi 443-760, Korea
}

\begin{abstract}
Trifoliate orange seed extracts (TSEs) were prepared from different solvents, water (TW), ethanol (TE), and n-hexane (TH), and assessed for their antimicrobial activities against six gram-negative food-bome pathogens (Escherichia coli KCTC 1039, Escherichia coli 0157:H7 ATCC 43895, Salmonella Enteritidis ATCC 3311, Salmonella Typhimurium KCCM 11862, Shigella sonnei KCTC 2518, and Vibrio parahaemolyticus ATCC 17802). Among the tested TSEs, TE and TH showed a slight inhibition activity on V. parahaemolyticus ATCC 17802, but a good growth inhibition activity on Sal. Typhimurium KCCM 11862. TH and TE showed steady growth inhibition activity with increasing growth time after $6 \mathrm{hr}$ when compared to the control $(p<0.05)$. From these results, we confimed the possibility of TH and TE as antimicrobial materials.
\end{abstract}

Key words: antimicrobial activity, growth inhibition activity, trifoliate orange seed extract

\section{INTRODUCTION}

In recent years, consumers are becoming more conscious of the nutritional value and safety of foods and ingredients. There is an increasing preference for natural foods and food ingredients because they are believed to be safer, healthier, and pose less hazardous risk than to their synthetic counterparts (1). Several natural ingredients have already been isolated from plant materials, such as oil seeds, cereal crops, vegetables, fruits, leaves, roots, spices, and herbs (2).

Citrus fruits, a major contributor to human diet, have received attention by researchers due to their multitude of bioactive compounds. Recent in vitro studies suggest these bioactive compounds have health-promoting properties and show potentials to be antioxidant, anti-proliferative, and antiviral agents, as well as preventing cardiovascular diseases (3). Citrus fruits are grown commercially in more than 140 countries around the world, and oranges, grapefruits, and lemons are considered to be the top three highly consumed fruits throughout the world (4). Among them, grapefruit seed extract (GSE) is a commercial product derived from the seeds and pulp of grapefruit (Citrus paradise Macf. Rutaceae). GSE is a natural extract that demonstrates effective broad-spectrum bactericidal, fungicidal, antiviral and anti-parasitic activities $(5,6)$. GSE is also environmentally safe without toxicity to humans or animals at effective concentrations. Ionescu et al. demonstrated that GSE performed as well as other antimicrobial agents tested on 770 strains of bacteria and 93 strains of fungi. On the other hand, only little information is available on studies relating to the antimicrobial activity of trifoliate orange (Poncirus trifoliate) and, more importantly, its seeds, which are a major byproduct with unknown significance. Based on this information, we focused on evaluating the bioactivity of the trifoliate orange seed extract (TSE). Health-promoting properties and the mechanism underlying the bioactive characteristics of TSE remain largely unknown when compared to GSE. This study is aimed at determining the health benefits of the well-utilized food byproducts, seeds, which can lead to economic benefits for the citrus processing industry, citrus growers, and the global society as a whole.

\section{MATERIALS AND METHODS}

\section{Preparation of TSEs}

Trifoliate orange was cultivated at Yeongcheon, Gyeongbuk, Korea and harvested in November, 2011. Dried trifoliate orange seeds were purchased from Cheongmyung Medicinal Herb Company (Gwangju, Korea). The trifoliate orange seeds were removed from foreign materials, including the peel, etc., by naked eye identification and then ground with an electronic grinder (Hanil Electronics Corp., Wonju, Korea). One hundred grams of the ground sample (moisture content; 10.61\%) was extracted with $1 \mathrm{~L}$ of distilled water, ethanol, or 
$n$-hexane. The extraction condition of ethanol and $n$-hexane was at room temperature for $8 \mathrm{hr}$, while the distilled water was kept in the $40^{\circ} \mathrm{C}$ incubator (Sanyo Electric Co. Ltd, Moriguchi, Japan) to maintain the temperature for $8 \mathrm{hr}$ with stirring, respectively. Each extracted sample was centrifuged at $6,000 \times g$ for $20 \mathrm{~min}$. The supernatant was concentrated with a rotary vacuum evaporator and then lyophilized, which was finally used as the extracted sample. The positive control used for antimicrobial activity testing was commercially available GSE (Esfood Co. Ltd., Pocheon, Korea).

\section{Bacterial strains tested and growth conditions}

Escherichia coli KCTC 1039, Escherichia coli O157: H7 ATCC 43895, Salmonella Enteritidis ATCC 3311, Salmonella Typhimurium KCCM 11862, Shigella sonnei KCTC 2518, and Vibrio parahaemolyticus ATCC 17802 were used as the gram-negative food-borne pathogens. Lactobacillus acidophilus IFO 3025 was the efficient bacteria used for testing the prebiotic potential of the test samples. The media and culture conditions for these strains are shown in Table 1. Stock cultures of these strains were activated in their appropriate media and conditions twice and then used to test for antimicrobial activity.

\section{Antimicrobial activities of TSEs using disc diffusion method on gram-negative food-bome pathogens}

Each test sample $(20 \mathrm{mg} / \mathrm{mL})$ of TW (trifoliate orange seed extracted by distilled water), TE (trifoliate orange seed extracted by ethanol), and TH (trifoliate orange seed extracted by $n$-hexane) for antimicrobial test was dissolved in distilled water, $75 \%$ dimethylsulfoxide (DMSO), and $100 \%$ DMSO, respectively. The samples were further filtrated with a membrane filter $(0.22 \mu \mathrm{m})$ before use. To assess the antimicrobial activity of TSEs on the test microorganisms' growth, paper disc agar diffusion method was performed. A $0.1 \mathrm{~mL}$ aliquot of the bacterial suspension at a cell density of $10^{6} \sim 10^{7} \mathrm{CFU} /$

Table 1. Bacterial strains tested and their growth conditions

\begin{tabular}{llc}
\hline \multicolumn{1}{c}{ Bacterial strains } & \multicolumn{1}{c}{ Media } & $\begin{array}{c}\text { Temperature } \\
\left({ }^{\circ} \mathrm{C}\right)\end{array}$ \\
\hline $\begin{array}{l}\text { Escherichia coli KCTC 1039 } \\
\text { Escherichia coli O157:H7 }\end{array}$ & $\begin{array}{l}\text { Nutrient agar } \\
\text { Tryptic soy }\end{array}$ & 37 \\
$\quad$ ATCC 43895 & $\begin{array}{c}\text { agar } \\
\text { Nutrient agar }\end{array}$ & 37 \\
$\begin{array}{l}\text { Salmonella Enteritidis } \\
\text { ATCC 3311 }\end{array}$ & Nutrient agar & 37 \\
$\begin{array}{l}\text { Salmonella Typhimurium } \\
\text { KCCM 11862 }\end{array}$ & \\
$\begin{array}{l}\text { Shigella sonnei KCTC 2518 } \\
\text { Vibrio parahaemolyticus } \\
\quad \text { ATCC 17802 }\end{array}$ & Nutrient agar & 37 \\
$\begin{array}{l}\text { Lactobacillus acidophilus agar } \\
\text { IFO 3025 }\end{array}$ & MRS agar & 37 \\
\hline
\end{tabular}

$\mathrm{mL}$ was spread on appropriate solid growth media for each food pathogen. After air-drying, a sterile $6 \mathrm{~mm}$ (diameter) paper disc was placed on the agar surface that had been inoculated with the test bacteria. Twenty microliter of each test sample and $20 \mu \mathrm{L}$ of distilled water, $75 \%$ DMSO, and $100 \%$ DMSO as negative control were added on the paper disc. The positive control disc was a GSE $(20 \mathrm{mg} / \mathrm{mL})$ filtrated membrane. All plates were then incubated at their respective optimal growth temperatures for 2 days under microaerophilic conditions. Inhibitory activity was measured (in $\mathrm{mm}$ ) as a diameter of the observed zones. All growth inhibition tests were replicated 3 times at $400 \mu \mathrm{g} / \mathrm{disc}$ and the antimicrobial activity was determined by assigning one of the following values based on the estimated diameter size of the zone of inhibition produced by each test sample: strong response $(+++)$, zone of inhibition diameter $>10 \mathrm{~mm}$; moderate response $(++)$, zone of inhibition diameter $8 \sim 10$ $\mathrm{mm}$; slight response $(+)$, zone of inhibition diameter $6 \sim$ $8 \mathrm{~mm}$; and no response (-), zone of inhibition diameter $6 \mathrm{~mm}$.

\section{Growth inhibition curve of TSEs on gram-negative food-bome pathogens}

Growth inhibition curves of TSEs on gram-negative food-borne pathogens were measured. First, we selected the test samples showing strong antimicrobial responses against food pathogens using the disc diffusion method and then determined the growth inhibition curve as a function of increasing time. Thirty milliliter of growth media, appropriate for each food pathogen growth, was added into a $100 \mathrm{~mL} \Delta$-flask and then autoclaved at $121^{\circ} \mathrm{C}$ for $15 \mathrm{~min}$. Three hundred microliter $(10 \mathrm{mg} / \mathrm{mL})$ of the test sample was filter sterilized via the membrane and the test pathogen at a concentration of $1 \%(\mathrm{v} / \mathrm{v}, 1 \sim 5$ $\times 10^{8} \mathrm{CFU} / \mathrm{mL}$ ) was added. Each flask was gently shaken and incubated in an incubator (IS-971R, Jeio Tech., Gimpo, Korea) under the respective optimal temperatures. The absorbance of each culture sample at increasing time points was measured by a UV-Visible Spectrophotometer (UV-2401PC, Shimadzu Corp., Kyoto, Japan) at $600 \mathrm{~nm}$.

\section{Prebiotic effects of TSEs on LAB}

Prebiotic effects of TSEs on Lb. acidophilus IFO 3025 were measured. Briefly, $30 \mathrm{~mL}$ of MRS broth for $L b$. acidophilus IFO 3025 was added in a $100 \mathrm{~mL} \Delta$-flask and then autoclaved at $121^{\circ} \mathrm{C}$ for $15 \mathrm{~min}$. Three hundred of microliter $(10 \mathrm{mg} / \mathrm{mL})$ of the test sample "sterilized" via the filter membrane and Lb. acidophilus IFO 3025 at a concentration of $1 \%\left(\mathrm{v} / \mathrm{v}, 1 \sim 5 \times 10^{8} \mathrm{CFU} / \mathrm{mL}\right)$ were added. Each flask was incubated at $37^{\circ} \mathrm{C}$ with gentle shaking (IS-971R, Jeio Tech.) and the absorbance was 
measured at $600 \mathrm{~nm}$ as previously described.

\section{Statistical analysis}

All values shown are the means of triplicate determinations. All statistical analyses were conducted using the Statistical Package for Social Sciences, version 12.0 (SPSS Inc., Chicago, IL, USA). The differences among samples were evaluated statistically by one-way analysis of variance (ANOVA) and Duncan's multiple tests. All data were evaluated at the $5 \%$ significance level using two-sided tests and are reported as the means \pm standard deviations.

\section{RESULTS}

\section{Antimicrobial activities of TSEs using disc diffusion method on gram-negative food-bome pathogens}

TSEs were made using distilled water, ethanol, and $n$-hexane. The yields of these 3 TSEs were as follows (Table 2): water extract (TW, 20.85\%) > ethanol extract $(\mathrm{TE}, 5.39 \%)>n$-hexane extract $(\mathrm{TH}, 0.44 \%)(\mathrm{p}<0.05)$. The TW yield was 3.89 times higher than that of the ethanol extract. The antimicrobial activity of TSEs prepared from different solvents was determined via the paper disc agar diffusion method against gram-negative food-borne pathogens (Table 3). TE showed a strong antimicrobial activity against Sh. sonnei KCTC 2518 and V. parahaemolyticus ATCC 17802, as did TH, which also showed strong antimicrobial activity against Sal. Typhimurium KCCM 11862. TW and negative control (distilled water, $75 \%$ DMSO, and 100\% DMSO) did not show any antimicrobial activity against the tested strains,

Table 2. Lyophilized powder yield of TSEs (trifoliate orange seed extracts) prepared from different solvents

\begin{tabular}{lccc}
\hline Sample $^{1)}$ & TW & TE & TH \\
\hline Yield (\%) & $20.85^{\mathrm{c}}$ & $5.39^{\mathrm{b}}$ & $0.44^{\mathrm{a}}$ \\
\hline
\end{tabular}

${ }^{1)} \mathrm{TW}$ : trifoliate orange seed extracted by water, TE: trifoliate orange seed extracted by ethanol, TH: trifoliate orange seed extracted by $n$-hexane. ${ }^{\mathrm{a}-\mathrm{c}}$ Significantly different at $\mathrm{p}<0.05$. whereas GW, the positive control, showed the strongest antimicrobial activity against all strains.

\section{Growth inhibition curve of TSEs on gram-negative food-bome pathogens}

The disk diffusion assay is a rapid and practical approach to screen a large number of potential antimicrobials; however, the method is limited by the diffusion rates of the active compounds in the agar media and does not account for the potential effect of a food matrix, which awaits further study for clarification. Based on the data, we selected the TSEs with antimicrobial activities and the pathogens they responded against: TE, TH, and GW, and Sh. sonnei KCTC 2518, Sal. Typhimurium KCCM 11862, and V. parahaemolyticus ATCC 17802. Namely, the inhibitory effects on the growth of the selected pathogens by TE, TH, and GW were determined as a function of time. For V. parahaemolyticus ATCC 17802, TE and TH treated samples showed minor steady inhibition activity compared with the control, especially at 60 and $123 \mathrm{hr}$, whereas GW treated samples showed similar inhibitory activity until $48 \mathrm{hr}$. However, proliferation was observed after $57 \mathrm{hr}$ in the GW treated sample when compared to the control (Fig. 1). For Sal. Typhimurium KCCM 11862, all tested samples demonstrated growth inhibition activity after $24 \mathrm{hr}$, from highest to lowest as follows: $\mathrm{GW}>\mathrm{TH}>\mathrm{TE}>$ control $(\mathrm{p}<$ 0.05 ). Interestingly, TH and TE showed steady growth inhibition activity with increasing growth time after 6 hr compared to the contrsol, whereas GW the growth inhibition activity was observed from the beginning of growth time $(\mathrm{p}<0.05)$. Only $\mathrm{GW}$ demonstrated growth inhibition activity against Sh. sonnei KCTC 2518. On the contrary, bacterial proliferation activity in $\mathrm{TH}$ and TE treated samples occurred after $12 \mathrm{hr}$ compared to the control, particularly at $24 \mathrm{hr}(\mathrm{p}<0.05)$.

\section{Prebiotic effects of TSEs on LAB}

$\mathrm{TE}, \mathrm{TH}$, and $\mathrm{GW}$ were examined for their probiotic effect, measured as the ability to increase the growth

Table 3. Antimicrobial activity of TSEs (trifoliate orange seed extracts) prepared from different solvents on gram-negative food-borne pathogens using disc diffusion method

\begin{tabular}{lcccc}
\hline \multirow{2}{*}{ Gram-negative foodborne pathogens } & \multicolumn{3}{c}{ Sample $^{1)}$} \\
\cline { 2 - 5 } & TW & TE & TH & GW \\
\hline Escherichia coli KCTC 1039 & - & + & + & +++ \\
Escherichia coli O157: H7 ATCC 43895 & - & + & + & ++ \\
Shigella sonnei KCTC 2518 & - & +++ & ++ & ++ \\
Salmonella Enteritidis ATCC 3311 & - & - & ++ & ++ \\
Salmonella Typhimurium KCCM 11862 & - & ++ & ++ & +++ \\
Vibrio parahaemolyticus ATCC 17802 & - & +++ & +++ & +++
\end{tabular}

${ }^{1)} \mathrm{TW}$ : trifoliate orange seed extracted by water, TE: trifoliate orange seed extracted by ethanol, TH: trifoliate orange seed extracted by $n$-hexane, GW: GSE (grape fruit seed extract) dissolve in water.

${ }^{2)}-$ : no inhibition $(6 \mathrm{~mm}),+$ : slight inhibition $(6 \sim 8 \mathrm{~mm}),++$ : moderate inhibition $(8 \sim 10 \mathrm{~mm}),+++$ : strong inhibition $(>10$ $\mathrm{mm})$. 

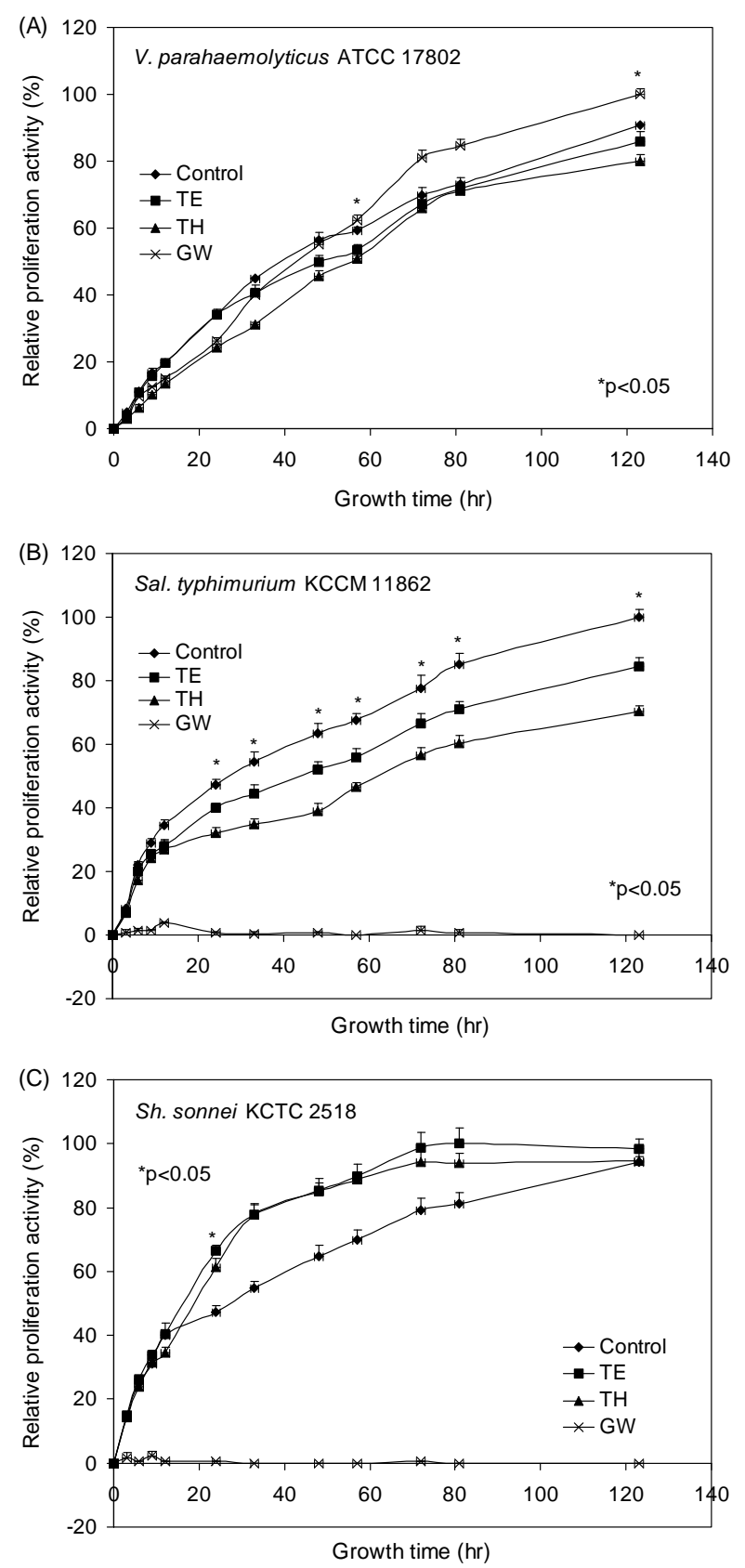

Fig. 1. Growth inhibition effects of TSEs (trifoliate orange seed extracts) prepared from different solvents on gram-negative food-borne pathogens $(\mathrm{A} \sim \mathrm{C})$. Control: No addition, TE: trifoliate orange seed extracted by ethanol, TH: trifoliate orange seed extracted by $n$-hexane, GW: GSE dissolved in water. ${ }^{*}$ Significantly different at $\mathrm{p}<0.05$ among the samples.

of the "good" bacteria. Lb. acidophius IFO 3025 was used as the representative beneficial probiotic bacteria. None of the tested samples demonstrated a prebiotic effect on $L b$. acidophius IFO 3025; interestingly, GW appeared to be slightly inhibitory during the initial growth time to $24 \mathrm{hr}$ (Fig. 2).

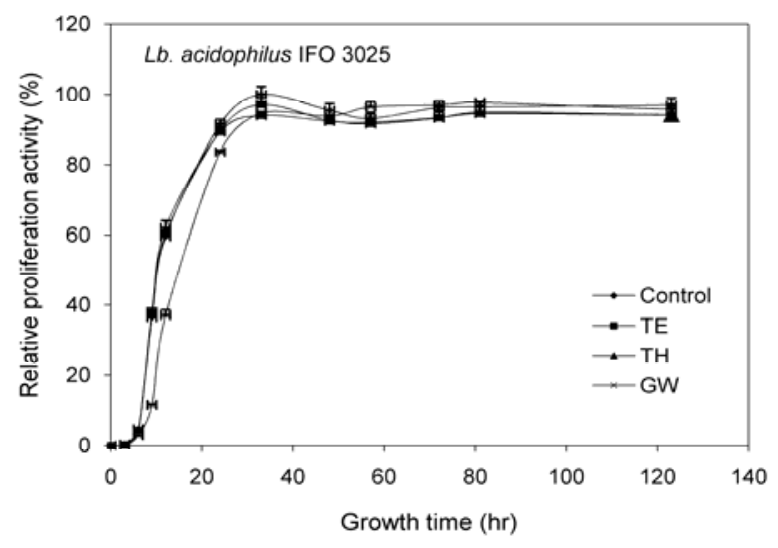

Fig. 2. Prebiotic effects of TSEs (trifoliate orange seed extracts) prepared from different solvents on the growth of Lactobacillus acidophilus IFO 3025. Control: No addition, TE: trifoliate orange seed extracted by ethanol, TH: trifoliate orange seed extracted by $n$-hexane, GW: GSE (grape fruit seed extract) dissolve in water.

\section{DISCUSSION}

A broad spectrum of microbial pathogens can contaminate human food and cause illnesses after they or their toxins are ingested (7). Consumption of raw foods has been suspected or contaminated worldwide as the most likely source of infection in diverse outbreaks which occurred during the last decade (8). The approaches that can be adopted in food preservation include: (a) aseptic handling and packaging, (b) mechanical removal of microorganisms by washing or filtration, (c) destruction of microorganisms by physical or chemical sanitization, and finally (d) inhibition of pathogens or saprophytes through environmental control achieved by adding synthetic chemical compounds (antimicrobial preservatives) such as trisodium phosphate, acidified calcium sulfate, organic acids (e.g., lactic, acetic) and acetyl pyridinium chloride with inhibitory or bactericidal/ fungicidal activity (9). Although approved for use in food processing in the last years, natural antimicrobials have attracted considerable attention due to the increasing consumer awareness on the aspects of food quality and safety.

Several methods have also been used to extend the storage life of green produce, such as high hydrostatic pressure, high intensity ultrasound and gamma irradiation. However, these treatments can also affect the sensory properties of food products, alter the structures of proteins or produce free radicals that affect the flavor of fruit (10). Therefore, there is a growing interest in the development of sanitizers with antimicrobial activities without toxicity in order to maintain the sensory quality and extend shelf-life of minimally processed vegetables and fruit. 
Citrus fruits and juices have long been recognized to contain secondary metabolites including antioxidants such as ascorbic acid, flavanones, phenolics and pectin that are important in human nutrition. Limonoids are secondary metabolites present in all citrus fruit tissues (11), but the efficient activities of their seeds have not been established. Citrus oils are complex mixtures of natural compounds (approximately 400 compounds) whose contents depend on specific citrus cultivar, extraction and separation methods. Unlike many of the exotic plant extracts that have been proposed as new antimicrobials (12), citrus oils have been a part of the human diet for hundreds of years and thus have been generally recognized as safe (GRAS) by the Food and Drug Administration (FDA). Individual citrus oil components have demonstrated antimicrobial activity against major food-borne pathogens (13).

In recent years, GSE has been shown to possess antibacterial, antiviral, antifungal and anti-parasitic properties (6). GSE contains large quantities of polyphenolic compounds, such as catechins, epicatechin, epocatechin3-O-gallate, and dimeric, trimeric and tetrameric procyanidins (14). These beneficial actions of GSE have partly been attributed to the antioxidative activity of citrus flavonoids, such as naringenin (15). The safety of GSE has been tested in several areas, with Heggers et al. showing that GSE was not detrimental to human fibroblast skin cells in vitro (16).

There is an increasing consumer demand for fresh, healthy, convenient and additive-free ready-to-eat vegetables that are safe and nutritious. These products may be whole or fresh-cut and are usually sealed in semi-permeable packages and stored at low temperatures, they can harbor large and diverse pathogenic populations of microorganisms, such as L. monocytogenes and Sal. spp. (17). Several cases of food-borne illnesses have been attributed to the consumption of fresh-cut vegetables or fruit (18) and Sal. spp. is amongst the most notable causative bacterial pathogens (19).

In conclusion, among the tested TSEs, TH has a robust growth inhibition activity against Sal. Typhimurium KCCM 11862, while TE has a slight growth inhibition activity against Sal. Typhimurium KCCM 11862. TE and TH demonstrated neither a prebiotic effect on $L b$. acidophius IFO 3025 nor a growth inhibition activity. Our data demonstrated that TSEs can be used as antimicrobial materials, instead of GSE. Thus, TSEs, as a food processing byproduct, can be a cost-friendly and safe antimicrobial product option.

Similar to most bioactive compounds, antimicrobial agents are chemically reactive species, which can cause considerable problems when embedded into a complex food system, such as altering physical stability or integrity of the food chemistry as well as degrading the biological activity of the bioactive compounds (20). This poses a concentration window requirement, high enough to inhibit microbial growth but low enough to minimally alter the qualitative properties of the product as imposed by food regulation. Taking into account the complexity of the TSEs mixtures and their variability in the chemical composition, further studies are highly warranted to test varying concentration ranges and the relevant food matrices in order to better characterize their potential as antimicrobials.

\section{REFERENCES}

1. Farag RS, Badei AZ, Heweij FM, El-Baroty GSA. 1986. Antioxidant activity of some spices essential oils on linoleic acid oxidation in aqueous media. $\mathrm{J}$ Am Oil Chem Soc 66: 792-799.

2. Gil MI, Tom'as-Barber'an FA, Hess-Pierce B, Holcroft DM, Kader AA. 2000. Antioxidant activity of pomegranate juice and its relationship with phenolic composition and processing. J Agric Food Chem 48: 4581-4589.

3. Roy A, Saraf S. 2006. Overview of significant bioactive triterpenes distributed in plants kingdom. Biol Pharm Bull 29: 191-201.

4. Spreen TH. 2003. Projections of world production and consumption of citrus to 2010. Fruit Proc 13: 378-384.

5. Coombe BG. 1989. The grape berry as a sink. Acta Hortic 239: $149-158$.

6. Ionescu G, Kiehl R, Wichmann-Kunz F, Williams CH, Bauml LM, Levine S. 1990. Oral citrus seed extract. J Orthomol Med 5: 230-238.

7. Tauxe RV. 2002. Emerging foodborne pathogens. Int $J$ Food Microbiol 78: 31-41.

8. Orden JA, Cid D, Ruiz-Santa-Quiteria JA, Garcia S, Martinez S, De la Fuente R. 2002. Verotoxin-producing Escherichia coli (VTEC) enteropatogenic E. coli (EPEC) and necrotoxigenic E. coli (NTEC) isolated from healthy cattle in Spain. J Appl Microbiol 93: 29-35.

9. Kemp GK, Aldrich ML, Waldroup AL. 2000. Acidified sodium chlorite antimicrobial treatment of broiler carcasses. J Food Prot 63: 1087-1092.

10. Vercet A, Lopez P, Burgos J. 1998. Free radical production by manothermosonication. Ultrasonics 36: 615618.

11. Tian Q, Miller EG, Ahmad H, Tang L, Patil BS. 2001. Differential inhibition of human cancer cell proliferation by citrus limonoids. Nutr Cancer 40: 180-184.

12. Dorman HJ, Deans SG. 2000. Antimicrobial agents from plants: antibacterial activity of plant volatile oils. J Appl Microbiol 88: 308-316.

13. Fisher K, Rowe C, Phillips CA. 2007. The survival of three strains of Arcobacter butzleri in the presence of lemon, orange and bergamot essential oils and their components in vitro and on food. Lett Appl Microbiol 44: 495-499.

14. Saito M, Hosoyama H, Ariga T, Kataoka S, Yamaji N. 1998. Antiulcer activity of grape seed extract and procyanidins. J Agric Food Chem 46: 1460-1464.

15. Shoko T, Soichi T, Megumi MM, Eri F, Jun K, Michiko W. 1999. Isolation and identification of an antibacterial 
compound from grape and its application to food. Biosci Biotechnol Agrochem 73: 125-128.

16. Heggers JP, Cottingham J, Gusman J, Reagor L, Mccoy L, Carino E, Cox R, Zhao JG. 2002. The effectiveness of processed grapefruit-seed extract as an antibacterial agent. I. Mechanism of action and in vitro toxicity. $J$ Altern Complement Med 8: 333-340.

17. Stine SW, Song I, Choi CY, Gerba CP. 2005. Effect of relative humidity on preharvest survival of bacterial and viral pathogens on the surface of cantaloupe, lettuce, and bell peppers. J Food Protect 68: 1352-1358.

18. Bharathi S, Ramesh MN, Varadaraj MC. 2001. Predicting the behavioural pattern of Escherichia coli in minimally processed vegetables. Food Control 12: 275-284.

19. Brackett R. 1999. Incidence, contributing factors, and control of bacterial pathogens in produce. Postharvest Biol Technol 15: 305-311.

20. McClements J. 2005. Food emulsions. In Principles, Practices and Techniques. 2nd ed. CRC Press, Boca Raton, FL, USA. p 83-124.

(Received June 21, 2012; Accepted August 15, 2012) 